\title{
Anticuerpos contra Chlamydophila en pacientes con infarto agudo del miocardio y riesgo coronario, y su relación con la muerte
}

Guadalupe G arcía-Elorriaga, Q BP, D ra en $C_{,}{ }^{(1)}$ Juan Jesús Sánchez-Barriga, M en $C_{,}{ }^{(2)}$ Marco Antonio Ramos-Corrales, MC, ${ }^{(2)}$ C ésar González-Bonilla, MSP, D r en C.(1)

\begin{abstract}
García-Elorriaga G, Sánchez-Barriga J, Ramos-Corrales MA, González-Bonilla C. Anticuerpos contra Chlamydophila en pacientes con infarto agudo del miocardio y riesgo coronario, y su relación con la muerte. Salud Publica Mex 2005;47:227-233.
\end{abstract}

\section{Resumen}

Objetivo. Determinar si los anticuerpos contra Chlamydophila pneumoniae en pacientes con infarto agudo del miocardio y factores de riesgo coronario se asocian con la muerte. Material y métodos. Se hizo un estudio observacional, prospectivo, transversal y comparativo. Se incluyeron en el estudio 100 sujetos que, entre 1999 y 2000, estuvieron hospitalizados en la Unidad Coronaria del Hospital de Especialidades del C entro Médico La Raza, del Instituto Mexicano del Seguro So cial. Se trataba de una muestra constituida por pacientes de ambos sexos, mayores de 18 años, con infarto agudo del miocardio y riesgo coronario. Mediante microinmuno fluorescencia indirecta se identificaron anticuerpos contra Chlamydophila pneumoniae, Chlamydophila psitacii y Chlamydia trachomatis. De entre los 100 sujetos, se eligieron al azar 33, a quienes se les determinaron anticuerpos contra Chlamydophila, no sólo durante su estancia en el hospital, sino tambien al salir de éste y a los tres meses de haber sufrido el infarto agudo del miocardio. Se calcularon las medias y las desviaciones geométricas estándares para los títulos de anticuerpos contra Chlamydophila, y se determinó la razón de momios y el intervalo de confianza al $95 \%$ entre los factores de riesgo coronario y la muerte. Resultados. Setenta por ciento de los pacientes de la muestra inicial presentaron anticuerpos contra Chlamydophila pneumoniae; no se identificaron anticuerpos contra Chlamydophila psitacii y Chlamydia trachomatis. No se observó una fuerza de aso ciación estadísticamente significativa con la muerte en pacientes con infarto agudo del miocardio y factores de riesgo coronario. De los 33 individuos de la submuestra, 25

\author{
García-Elorriaga G, Sánchez-Barriga JJ, \\ Ramos-Corrales MA, González-Bonilla C. \\ Antibodies against Chlamydophila \\ in patients with acute myocardial infarction \\ and coronary risk and their association with mortality. \\ Salud Publica Mex 2005;47:227-233.
}

\section{A bstract}

Objective.The primary aim of this study was to determine whether antibo dies against Chlamydophila pneumoniae in patients with acute myocardial infarction (AMI) and coronary risk factors are associated with death. Material and Methods. A cross-sectional study was conducted among 100 patients hospitalized in the Coronary Unit of C entro Medico La Raza H ospital of the Mexican Institute of Social Security, between 1999 and 2000. Subjects were males and females older than 18 years, diagnosed with AMI and coronary risk.A ntibodies against $C$ hlamydo phila pneumoniae, Chlamydophila psitacii and Chlamydia trachomatis were measured using an indirect microinmunofluorescence assay. In addition, blood samples from 33 patients from the original group were taken when the patients were discharged from the hospital, and 3 months after their myocardial infarction. Data analysis consisted of geometric means and standard deviations as well as odds ratios with $95 \%$ confidence intervals. Results. Seventy percent of patients presented antibodies against Chlamydophila pneumoniae. Antibodies against Chlamydophila psitacii and Chlamydia trachomatis were not identified. No statistically significant association was found between antibodies and death in these patients with coronary risk factors and AMI. In the subgroup of 33 individuals 25 had antibodies against Chlamydophila pneumoniae and in $83 \%$ of them antibodies decreased three months after the AMI event. Conclusions Even though patients with coronary risk factors and $A M I$ had an increased seropositivity for Chlamydophila pneumoniae it was not significantly associated with death.

(1) Unidad de Investigación en Inmunología e Infectología, Hospital de Infectología del Centro Médico N acional La Raza, Instituto Mexicano del Seguro Social (IMSS). México, DF, México.

(2) Unidad Coronaria del Hospital de Especialidades del Centro Médico N acional La Raza. IMSS. México, DF, México.

Fecha de recibido: 30 de agosto de 2004 - Fecha de aprobado: 28 de marzo de 2005

Solicitud de sobretiros: Dra. Guadalupe García Elorriaga. Hospital de Infectología, Centro Médico N acional La Raza, IMSS. Avenida Jacarandas esquina Vallejo sn., colonia la Raza. 0200 México, DF, México.

Correo electrónico: gelorriaga@ prodigy.web.net.mx (o prodigiweb) 
presentaron anticuerpos contra Chlamydophila pneumoniae, y en $83 \%$ de estos últimos casos, se registró un descenso de dichos anticuerpos a los tres meses de haberse presentado el infarto agudo del miocardio. Conclusiones. $A$ pesar de que en pacientes con infarto agudo del miocardio y riesgo coronario se presentó un incremento en la frecuencia de seropositividad a Chlamydophila pneumoniae, no se observó una fuerza de asociación estadísticamente significativa de ello con la muerte.

Palabras clave: Chlamydophila pneumoniae; Chlamydophila psitacii; Chlamydia trachomatis; infarto agudo del miocardio; coronario patia; factores de riesgo; México
Key words: Chlamydophila pneumoniae; Chlamydophila psitacii; Chlamydia trachomatis; acute myocardial infarction; coronary disease; risk factors; Mexico
$L$ a aterosclerosis produce cardiopatía isquémica y embolia cerebral, causas importantes de morbilidad y mortalidad. La diabetes, hipertensión arterial sistémica, hipercolesterolemia y la obesidad, entre otros, son factores de riesgo que aumentan la probabilidad de presentar ateroesclerosis. Sin embargo, en la actualidad se están estudiando otros factores que pueden estar implicados en esta patología. Existe evidencia que sugiere que las infecciones también juegan un papel importante en el desarrollo de ateroesclerosis coronaria, una idea propuesta por Sir William Osler desde el comienzo de este siglo. ${ }^{1}$

La seropositividad hacia Chlamydophila pneumoniae ha sido significativamente más frecuente entre pacientes con infarto agudo del miocardio (IAM) en comparación con personas sanas. ${ }^{2}$ La serología positiva hacia C. pneumoniae se ha asociado con cardiopatía isquémica, después del ajuste para el efecto de los factores de riesgo conocidos (edad, tabaquismo, hipertensión arterial sistémica, proporción de lipoproteínas de alta densidad [HDL] y colesterol total). La presencia de complejos inmunes específicos de C. pneumoniae y un título elevado de anticuerpos IgA de 3 a 6 meses se asociaron con un riesgo mayor de dos veces para el desarrollo de cardiopatía isquémica. ${ }^{3}$ En un estudio de seguimiento de siete años a sujetos diabéticos y no diabéticos ${ }^{4}$ se observó que la seropositividad hacia C. pneumoniae se asoció con eventos futuros de cardiopatía isquémica en sujetos no diabéticos. Dos estudios han encontrado específicamente la asociación de serología positiva hacia C. pneumoniae en fumadores. ${ }^{5,6}$

Se ha observado una asociación entre anticuerpos IgA hacia C. pneumoniae y cardiopatía isquémica. La IgA es un marcador de infección crónica ${ }^{7,8}$ y riesgo subsecuente de muerte en pacientes con cardiopatía isquémica. ${ }^{9}$ Para determinar si la infección crónica tiene un papel importante en el inicio o progresión de la enfermedad, se han llevado a cabo estudios clínicos con tratamiento antibiótico en humanos para prevenir o mejorar el pronóstico de las enfermedades ateros- cleróticas por la implicación que pudiera tener $C$. pneumoniae en el proceso de ateroesclerosis. La administración de azitromicina disminuye el riesgo de eventos cardiovasculares en pacientes con infarto agudo del miocardio. ${ }^{10}$

El cultivo y aislamiento de $C$. pneumoniae es difícil y se desconoce su sensibilidad, por lo que son poco accesibles a los laboratorios de rutina. Si bien la reacción en cadena de la polimerasa (PCR) ha demostrado ser útil para la identificación de la bacteria, requiere de entrenamiento especial y personal experimentado, por lo que no es práctica. Por lo tanto, la serología es la herramienta más frecuentemente utilizada para el diagnóstico de rutina de la infección por $C$. pneumoniae. Actualmente, el estándar de oro en serología de C. pneumoniae es la prueba de microinmunofluorescencia (MIF). ${ }^{11,12}$ Hasta la fecha, se ha probado que el uso de MIF con antígeno de C. pneumoniae (TWAR) es el método más sensible y específico para el diagnóstico de infección. A diferencia de otras especies de Chlamydia, solamente se ha reconocido un serovar en el sistema MIF y prácticamente no existe la reacción cruzada con dichas especies. ${ }^{13}$

En un trabajo realizado por nuestro grupo, ${ }^{14}$ encontramos una prevalencia de anticuerpos de tipo IgG hacia C. pneumoniae de $94.3 \%$ (66/70) en pacientes con cardiopatía isquémica, y de ellos $42.8 \%$ tuvo IAM. Sin embargo, algunos estudios no han encontrado dicha asociación. $^{15}$

Por la relación que probablemente tiene $C$. pneumoniae con la ateroesclerosis en pacientes con IAM, ${ }^{16}$ es importante determinar si la infección por C. pneumoniae está asociada con la muerte de pacientes con diabetes mellitus tipo 2, hipertensión arterial sistémica, tabaquismo y con edad $>40$ años.

\section{Material y métodos}

Durante 1999 se incluyeron al estudio 100 pacientes de uno y otro sexo, mayores de 18 años con IAM que 
ingresaron de forma consecutiva a la Unidad Coronaria del Hospital de Especialidades del Centro Médico Nacional La Raza del Instituto Mexicano del Seguro Social. Todos los pacientes cumplieron con los criterios para infarto del miocardio. ${ }^{17}$ Se tomó una muestra sanguínea en el momento de su ingreso para determinar los títulos de IgG, IgM e IgA, por medio de MIF. Se les realizó historia clínica, electrocardiograma, a su ingreso, y posteriormente una vez al día durante su estancia hospitalaria. Se determinó creatinfosfoquinasa y su fracción MB, ecocardiograma y gammagrafía cardiaca con pirofosfato de tecnecio ( ${ }^{99 \mathrm{~m}}$ Tc-PYP). Los pacientes aceptaron y firmaron la carta de consentimiento informado para participar en el estudio. El protocolo fue aprobado por los Comités de Etica y de Investigación del Hospital.

\section{Factores de riesgo}

Se consideró tabaquismo en aquellos pacientes que tenían el antecedente de ser fumadores habituales; hipertensión arterial, cuando tenían el antecedente o habían presentado en tres ocasiones distintas, presión arterial $\geq 140 / 90 \mathrm{~mm}$ de $\mathrm{Hg}$ o tenían tratamiento antihipertensivo; hipercolesterolemia, cuando presentaban el antecedente de colesterol sérico $\geq 200 \mathrm{mg} / \mathrm{dl}$ o estaban bajo tratamiento para la hipercolesterolemia; diabetes mellitus, cuando las cifras de glucosa sérica en ayuno fueron $\geq 126 \mathrm{mg} / \mathrm{dl}$ en dos ocasiones, o tenían tratamiento para la diabetes mellitus, y obesidad, cuando se obtuvo un índice de masa corporal (IMC= peso $\mathrm{kg} /$ altura $\left.\mathrm{m}^{2}\right) \geq 30 .^{18}$

Se tomaron muestras sanguíneas a todos los pacientes y mediante centrifugación a $3500 \mathrm{rpm}$ se obtuvo el suero, que se refrigeró a $-20^{\circ} \mathrm{C}$ hasta su análisis mediante MIF, con el fin de identificar los anticuerpos contra $C$. pneumoniae. De los 100 pacientes, en 33 escogidos al azar también se determinó $C$. pneumoniae al alta hospitalaria y a los tres meses de evolución del IAM.

\section{Principio de la prueba \\ de microinmunofluorescencia indirecta \\ y análisis serológico}

El ensayo se hizo de acuerdo con las recomendaciones del productor (Cat. No. 6108380, Labsystems). Esta prueba detecta anticuerpos $\operatorname{IgG} / \operatorname{IgM} / \operatorname{IgA}$ contra $C$. pneumoniae de manera indirecta usando isotiocianato de fluoresceína (FITC) y azul de Evans colorante de contraste. Las laminillas se leyeron en un microscopio UV (Olympus- BX40), con un lente de inmersión de 100X y un lente ocular de 10X (aumento total 1000X), por el mismo investigador de manera ciega. El título final se consideró como la dilución más alta del suero que demostró fluorescencia definida y se expresó como el recíproco de la dilución del suero. El recíproco del título de $\operatorname{IgG} \geq 16$ se usó como el límite inferior para serología positiva, ${ }^{19}$ se consideró un título bajo de $16-32$, y positivo alto $\geq 64 .{ }^{20}$ Los títulos de $\operatorname{IgG} \geq 64$ contra C. trachomatis y C. psittaci se consideraron como positivos. ${ }^{21}$

\section{A nálisis estadístico}

Se calcularon las medias geométricas y las desviaciones geométricas estándares para los títulos de anticuerpos contra Chlamydophila. Se utilizó la distribución normal, pero modificada, en virtud de que el análisis de los resultados está en función de un factor de dilución, por lo que el análisis estadístico fue el de la distribución normal adaptada a logaritmos de base 2. Los resultados obtenidos se contrastaron con la $t$ de Student para muestras independientes.

Para determinar la razón de momios, intervalo de confianza a 95\% (IC 95\%), y el valor de $p$, entre los pacientes con hipertensión arterial sistémica, diabetes mellitus 2, hipercolesterolemia, tabaquismo, sexo, edad $>40$ años y obesidad, con anticuerpos IgG contra $C$. pneumoniae, y la muerte, se utilizó el programa informático Epi Info ${ }^{\mathrm{TM}} 2000$.

\section{Resultados}

Se incluyeron 100 pacientes que ingresaron con IAM a la Unidad Coronaria del Hospital de Especialidades del Centro Médico Nacional La Raza. Hubo 81 hombres y 19 mujeres con edad promedio de $58 \pm 11$ años: $96 \%$ fueron mayores de 40 años de edad, $38 \%$ presentó diabetes mellitus tipo 2, 34\% hipertensión arterial sistémica y $10 \%$ hipercolesterolemia; $50 \%$ tabaquismo y $29 \%$ tenía obesidad. En ningún paciente fue posible demostrar anticuerpos IgM contra alguna de las tres especies de Chlamydophila, lo que sugiere que ninguno cursaba con infección reciente por esta bacteria. El 70\% de los enfermos presentaron anticuerpos contra $C$. pneumoniae y ningún paciente con IAM presentó anticuerpos contra las otras dos especies de Chlamydia. Se observó que las mujeres presentaron títulos de $\operatorname{IgG}$ contra C. pneumoniae más bajos que los hombres. Catorce pacientes del sexo masculino presentaron título de 1:32, en tanto que sólo dos mujeres presentaron este título $(p<0.05)$. Trece hombres tuvieron un título 1:64 en comparación con sólo una mujer que presentó el mismo título (datos no mostrados).

Doce pacientes fallecieron en los primeros 15 días de hospitalización (cuadro I), de estos pacientes uno 


\section{Cuadro I \\ Características de los pacientes con infarto agudo del miocardio, incluidos en el estudio SOBRE COMPORTAMIENTO SEROLÓGICO DE CLAMIDIAS Y SU RELACIÓN CON FACTORES DE RIESGO CORONARIO. Centro Médico Nacional la Raza, Instituto Mexicano del Seguro Social, 1999-2000}

\begin{tabular}{|c|c|c|c|c|}
\hline Variable & $\begin{array}{c}\text { Todos } \\
\text { los pacientes } \\
n=100\end{array}$ & $\begin{array}{c}\text { Pacientes con anticuerpos } \\
\text { contra C. pneumoniae } \\
n=70\end{array}$ & $\begin{array}{l}\text { Pacientes sin anticuerpos } \\
\text { contra C. pneumoniae } \\
n=30\end{array}$ & $p$ \\
\hline Hombres/mujeres & $81(81 \%) / 19(19 \%)$ & $58(82 \%) / 12(17 \%)$ & $23(76 \%) / 7(23 \%)$ & $0.46^{\ddagger}$ \\
\hline$\overline{\text { Edad (años) }}$ & $58 \pm 11$ & $58 \pm 11$ & $57 \pm 10^{*}$ & $0.44^{\S}$ \\
\hline Edad mayor de 40 años & $96(96 \%)$ & $68(97 \%)$ & $28(93 \%)$ & $0.37^{\ddagger}$ \\
\hline Hipertensión arterial sistémica & $34(34 \%)$ & $24(34 \%)$ & $10(33 \%)$ & $0.92^{\ddagger}$ \\
\hline Diabetes mellitus tipo 2 & $38(38 \%)$ & $27(38 \%)$ & $11(36 \%)$ & $0.85^{\ddagger}$ \\
\hline Hipercolesterolemia & $10(10 \%)$ & $7(10 \%)$ & $3(10 \%)$ & $1^{\ddagger}$ \\
\hline Tabaquismo & $50(50 \%)$ & $35(50 \%)$ & $15(50 \%)$ & $1^{\ddagger}$ \\
\hline O besidad & $29(29 \%)$ & $16(22 \%)$ & $13(43 \%)$ & $0.03^{\ddagger}$ \\
\hline Infarto anterior extenso & $19(19 \%)$ & $13(18 \%)$ & $6(20 \%)$ & $0.86^{\ddagger}$ \\
\hline Arritmias & $40(40 \%)$ & $29(41 \%)$ & $11(36 \%)$ & $0.89 \ddagger$ \\
\hline Insuficiencia cardiaca & $16(16 \%)$ & $12(17 \%)$ & $4(13 \%)$ & $0.63^{\ddagger}$ \\
\hline Comunicación interventricular & $3(3 \%)$ & $2(2.8 \%)$ & $1(3.3 \%)$ & $0.89^{\ddagger}$ \\
\hline $\begin{array}{l}\text { Muerte } \\
* \bar{X} \pm D E \\
\ddagger \chi^{2} \text { para determinar diferencia } \\
\S \mathrm{t} \text { para determinar diferencias }\end{array}$ & $\begin{array}{l}\qquad 12(12 \%) \\
\text { ariables categóricas } \\
\text { iables numéricas }\end{array}$ & $11(15 \%)$ & $1(3.3 \%)$ & $0.08^{\ddagger}$ \\
\hline
\end{tabular}

no presentó anticuerpos contra C. pneumoniae. De los 11, seis fueron positivos con título 1:16, dos 1:32; dos 1:64 y uno 1:256. En 25 de los 33 pacientes con tres muestras de suero se presentaron anticuerpos contra C. pneumoniae en el momento del IAM y en $83 \%$ de éstos hubo un descenso a los tres meses; en los tres pacientes restantes los títulos se incrementaron posterior al infarto agudo del miocardio (figura 1). De los 12 pacientes que fallecieron, seis fueron hombres $(7.4 \%$ de la población masculina total estudiada) y seis mujeres (31\%). En todos ellos los títulos de IgG contra $C$. pneumoniae fueron positivos. Los títulos de IgM fueron negativos en los 100 pacientes con IAM.

Cuando se analizó la fuerza de asociación con la muerte en pacientes con IAM, hipertensos, diabéticos, fumadores, obesos y con edad mayor de 40 años, portadores de anticuerpos contra C. pneumoniae, no se observo una asociación estadísticamente significativa con la mortalidad (cuadro II). De los 33 pacientes a los que se les determinó anticuerpos contra C. pneumoniae al ingreso, al alta hospitalaria y a los tres meses de evolución del IAM, se observó (figura 1) que el comportamiento es irregular; sin embargo, cuando se hizo la suma del total de las diluciones positivas $(\geq 1: 16)$, en

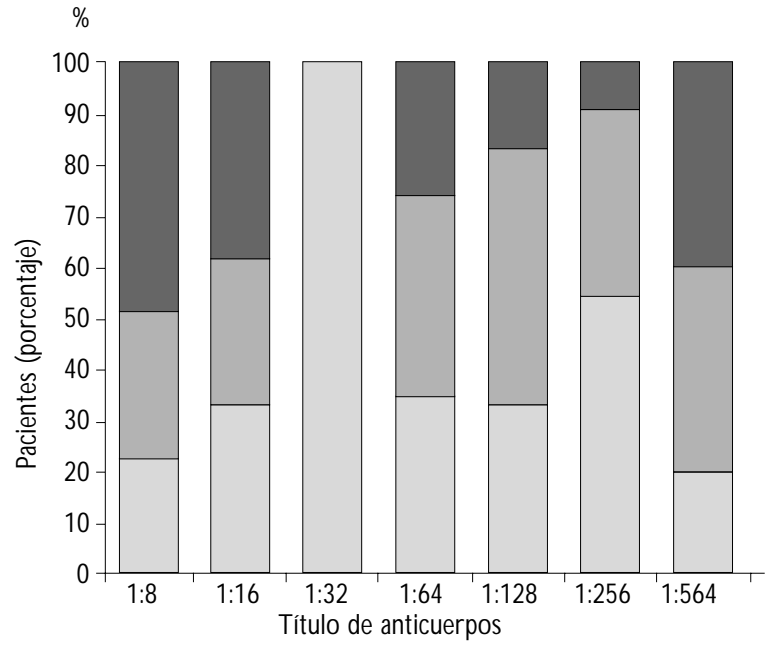

A los tres meses

Al egreso

Al ingreso

Figura 1.Título de anticuerpos contra ChlamydoPHILA PNEUM ONIAE AL INGRESO, AL EGRESO HOSPITALARIO Y A LOS TRES MESES, EN 33 PACIENTES CON INFARTO AGUDO del miocardio. Centro Médico Nacional la Raza, Instituto Mexicano del Seguro Social, 1999-2000 
Cuadro II

Asociación ENTRE FACTORES DE RIESGo CORONARIO ESTUDIADOS CON MUERTE, EN PACIENTES CON INFARTO AGUDO AL MIOCARDIO Y ANTICUERPOS CONTRA Chlamydophila pneumoniae. Centro Médico Nacional La Raza, Instituto Mexicano del Seguro Social, 1999-2000

\begin{tabular}{llll} 
& \multicolumn{3}{c}{ Muerte } \\
\cline { 2 - 4 } Factores de riesgo coronario & $\mathrm{RM}$ & IC $95 \%$ & $\mathrm{p}$ \\
Hipertensión arterial sistémica & 2.25 & $0.31-16.9$ & 0.38 \\
\hline Diabetes mellitus tipo 2 & 2.86 & $0.26-71.7$ & 0.30 \\
\hline Tabaquismo & 2.17 & $0.20-54$ & 0.49 \\
\hline O besidad & 2.77 & $0.20-79$ & 0.39 \\
\hline Edad mayor de 40 años & 5.02 & $0.61-109$ & 0.09
\end{tabular}

Cuadro III

Determinación de anticuerpos anti-C hlam ydo Phila PNEUMONIAE AL INGRESO, AL EGRESO HOSPITALARIO Y A LOS TRES MESES, EN 33 PACIENTES CON INFARTO agudo del miocardio. Centro Médico Nacional La Raza, Instituto Mexicano del Seguro Social, 1999-2000

\begin{tabular}{lccc} 
Título & $\begin{array}{c}\text { Ingreso } \\
\text { al hospital }\end{array}$ & $\begin{array}{c}\text { Egreso } \\
\text { del hospital }\end{array}$ & $\begin{array}{c}\text { Tres meses de evolución } \\
\text { del infarto del miocardio }\end{array}$ \\
$1: 8$ & 7 & 9 & 15 \\
\hline Total negativos & 7 & 9 & 15 \\
$1: 16$ & 7 & 6 & 8 \\
\hline $1: 32$ & 2 & 0 & 0 \\
\hline $1: 64$ & 8 & 9 & 6 \\
\hline $1: 128$ & 2 & 3 & 1 \\
\hline $1: 256$ & 6 & 4 & 1 \\
\hline $1: 564$ & 1 & 2 & 2 \\
\hline Total positivos & 26 & 24 & 18
\end{tabular}

Fecha en que se realizó el estudio: 1999-2000

Lugar en donde se realizó el estudio:

Unidad Coronaria del Hospital de Especialidades del Centro Médico La Raza

Unidad de Investigación en Enfermedades Infecciosas. Hospital de Infectología del Centro Médico La Raza

los tres periodos mencionados se observó que la positividad de los anticuerpos fue mayor al ingreso, y fue disminuyendo al egreso hospitalario y a los tres meses de evolución del IAM (cuadro III, figura 1).

\section{Discusión}

En nuestro trabajo 70\% de los pacientes con IAM demostró anticuerpos contra el lipopolisacárido de Chlamydophila; en otros estudios se ha documentado una prevalencia de $55.2 \%, 80.4 \%, 74.0 \%$ y $73.2 \%, 22,23$ de acuerdo con lo reportado en la literatura, nuestros resultados no tienen una variación importante en la prevalencia de IgG. En un trabajo previo ${ }^{14}$ encontramos una prevalencia de anticuerpos positivos contra $C$. pneumoniae de $94.3 \%$ en una muestra de 70 pacientes con cardiopatía isquémica, 30 de los cuales presentaron IAM; es posible que la prevalencia de anticuerpos positivos contra C. pneumoniae en el presente estudio sea menor porque estudiamos un mayor número de pacientes con IAM $(n=100)$.

El comportamiento serológico de IgG contra $C$. pneumoniae en los 33 pacientes con IAM fue el siguiente: la media geométrica $(\mathrm{Xg}), \pm$ la desviación geométrica estándar $\left(\sigma_{g}\right)$, del inverso del título en el momento del infarto fue de $42.2 \pm 7.6$, al egreso del hospital de $40.6 \pm 7.6$ y de $21.6 \pm 7.5$ a los tres meses; el descenso de los títulos obtenidos resultó estadísticamente significativo. Por otro lado, en la figura 2 se comparan los títulos de anticuerpos contra Chlamydophila pneumoniae de todos los pacientes (barras negras) en relación con aquellos que tuvieron títulos positivos (1:16 o mayo-

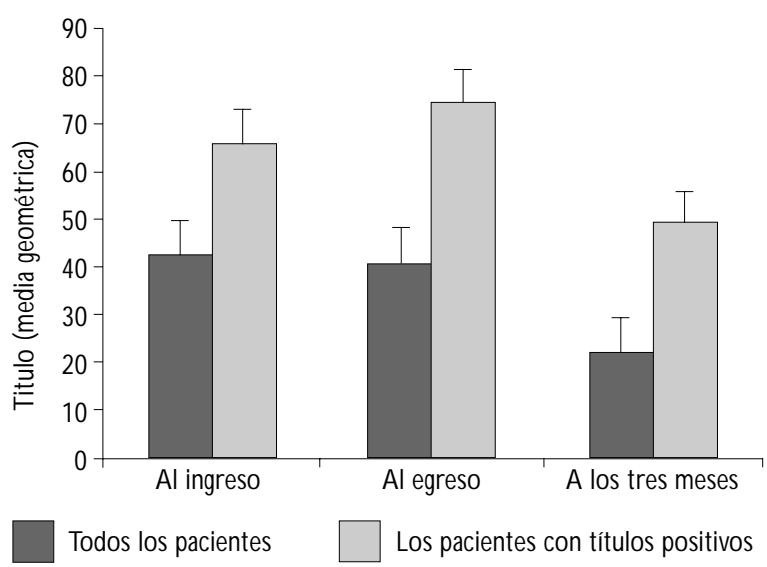

Figura 2. Medias geométricas del títUlo de anticuerPOS CONTRA ChLAM ydo Phila PNEUMONIAE EN 33 PACIENTES CON INFARTO AGUDO DEL MIOCARDIO AL INGRESO, AL EGRESO DEL HOSPITAL Y TRES MESES DESPUÉS DEL INFARTO. Centro Médico Nacional la Raza, Instituto MexiCano del Seguro Social, 1999-2000 
res) al momento de la primera toma (barras grises); puede observarse que en estos pacientes los títulos ascendieron al momento del egreso del hospital y descendieron tres meses después. Este comportamiento serológico sugiere una respuesta de memoria a antígenos de C. pneumoniae, que puede estar dada por la liberación de antígenos de C. pneumoniae durante la ruptura de la placa ateroesclerosa que condiciona el $\mathrm{IAM}_{,}{ }^{24}$ ya que se ha encontrado C. pneumoniae en placas ateroesclerosas en las arterias coronarias, ${ }^{25,26}$ esta respuesta inmunológica se puede producir cuando ya existen anticuerpos de memoria circulantes contra $C$. pneumoniae por infección previa.

De los 12 pacientes que fallecieron, 11 presentaron anticuerpos de tipo IgG hacia C. pneumoniae. De la población total por sexo el porcentaje de fallecimientos fue menor en hombres $(7.4 \%)$ en relación con las mujeres (31\%). Cuando determinamos la fuerza de asociación entre los anticuerpos contra Chlamydophila y los factores de riesgo ya conocidos para infarto y muerte, no se observó una fuerza de asociación estadísticamente significativa en pacientes con diabetes mellitus tipo 2, fumadores, obesos, hipertensos y en los pacientes mayores de 40 años (cuadro II). Esto sugiere que en los pacientes con anticuerpos positivos contra C. pneumoniae no se incrementa el riesgo de muerte, lo referido anteriormente coincide con algunos estudios. Strachan $\mathrm{DP}^{8}$ observó en 642 hombres (36.2\%), de un total de 1 773 , que no existe asociación entre la IgG y la muerte en pacientes con IAM, pero en los enfermos con anticuerpos IgA positivos (dilución 1:16) contra C. pneumoniae se identificó un incremento de la probabilidad para muerte (RM 1.83, IC 95\% 1.17-2.85). Wald $\mathrm{NJ}^{27}$ en un estudio de casos y controles anidados en una cohorte (647 casos y 1294 controles), determinó la razón de momios para $\operatorname{IgG}$ e $\operatorname{IgA}$, para muerte en pacientes que fallecieron por IAM; encontró para la IgG una (RM de 1.26 con IC 95\% 0.95-1.68, y para la IgA, RM 1.09, IC 95\% 0.82-1.43; en este estudio no hubo una fuerza de asociación estadísticamente significativa entre los anticuerpos IgG e IgA contra C. pneumoniae, y la muerte en pacientes con IAM. En otro estudio de casos y controles (casos $n=213$ y controles $n=405$ ), en una cohorte de pacientes $\geq 65$ años, Siscovick DS y colaboradores ${ }^{28}$ encontraron una fuerza de asociación estadísticamente significativa entre anticuerpos IgG contra C. pneumoniae y la muerte, en pacientes con IAM con títulos altos (1:1024), (RM 2.2 IC 95\% 1.1-4.4); mientras que en los pacientes con títulos bajos (£1:512) no se observó fuerza de asociación significativa (RM 1.1, IC 95\% 1.1, 0.7-1.8).

Los resultados obtenidos se parecen a los observados en otros estudios; sin embargo, son pocos los tra- bajos publicados que se relacionan con el tema, por lo que consideramos necesarios más estudios para establecer si existe relación entre los anticuerpos IgG contra C. pneumoniae y la muerte en pacientes con IAM.

\section{Conclusiones}

A pesar de que el IAM se observó con una frecuencia incrementada de $70 \%$ de seropositividad hacia C. pneumoniae, no se presentó una fuerza de asociación estadísticamente significativa con la muerte en pacientes con IAM, hipertensos, diabéticos, fumadores, obesos y con edad mayor de 40 años.

\section{Referencias}

1. 0 sler W. D iseases of the arteries. En: 0 sler W, ed. Modern medicine: Its theory and practice. Filadelfia: Lea \& Febiger; 1908: 426-447. 2. Basinkevich AB, Shakhnovich RM, Martynova VR, Kolkova N I, Rakovskaya IV, Karazhas N V et al. [Role of Chlamydia, mycoplasma and cytomegalovirus infection in the development of coronary artery disease]. Kardiologiia 2003;43:4-9.

3. Saikku P, Leinonen M,Tenkanen L, Linnanmaki E, Ekman MR, Manninen $\checkmark$ et al. Chronic Chlamydia pneumoniae infection as a risk factor for coronary heart disease in the Helsinki Heart Study. Ann Intern Med 1992;116:273-278.

4. Miettinen $H$, Lehto S, Saikku P, H affner SM, Ronnemaa T, Pyorala $K$ et al.Association of Chlamydia pneumoniae and acute coronary heart disease events in non-insulin dependent diabetic and non-diabetic subjects in Finland. Eur Heart J 1996;17:682-688.

5. Hahn DL, Golubjatnikov R. Smoking is a potential confounder of the Chlamydia pneumoniae-coronary artery disease association. Arterioscler Thromb 1992;12:945-947.

6. Karvonen M, Tuomilehto J, Pitkaniemi J, N aukkarinen A, Saikku P. Importance of smoking for Chlamydia pneumoniae seropositivity. Int J Epidemiol 1994;23:1315-1321.

7.W olf SC, Mayer 0 , Jurgens S, Vonthein R, Schultze G, Risler T et al. Chlamydia pneumoniae IgA seropositivity is associated with increased risk for atherosclerotic vascular disease, myocardial infarction and stroke in dialysis patients. Clin N ephrol 2003;59:273-279.

8. Strachan DP, Carrington D, Mendall MA, Ballam L, Morris J, Butland BK et al. Relation of Chlamydia pneumoniae serology to mortality and incidence of ischaemic heart disease over 13 years in the caerphilly prospective heart disease study. BMJ 1999;318:1035-1039.

9. Haubitz $M$, Votsch $K, W$ oywodt $A, N$ ashan $B, G$ roh A, Haller $H$ et al. Serologic evidence of Chlamydia pneumoniae infection as a long-term predictor of cardiovascular death in renal transplant recipients. Transplantation 2004;77:1517-1521.

10. Stone AF, Mendall MA, Kaski JC, Edger TM, Risley P, Poloniecki J et al. Effect of treatment for Chlamydia pneumoniae and Helicobacter pylori on markers of inflammation and cardiac events in patients with acute coronary syndromes: South Thames Trial of Antibiotics in Myocardial Infarction and Unstable Angina (STAMIN A). C irculation 2002;106:12191223.

11. Cosentini R, Blasi F, Raccanelli R, Rossi S,Arosio C, Tarsia P et al. Severe community-acquired pneumonia:A possible role for Chlamydia pneumoniae. Respiration 1996;63:61-65. 
12. Kauppinen MT, Saikku P, Kujala P, Herva E, Syrjala H. Clinical picture of community-acquired Chlamydia pneumoniae pneumonia requiring hospital treatment:A comparison between chlamydial and pneumococcal pneumonia. Thorax 1996;51:185-189.

13.W ang S.The microimmunofluorescence test for Chlamydia pneumoniae infection:Technique and interpretation.J Infect $D$ is 2000;181 Suppl 3:S4215.

14. García-Elorriaga GL, Calderón-A bbo M, G onzález-Bonilla CR. Asociación entre enfermedad cardiovascular y anticuerpos contra Chlamydia pneumoniae. Salud Publica Mex 2002;44:243-246.

15. Bloemenkamp D G, Mali W P,Visseren FL, van der GraafY. Metaanalysis of sero-epidemiologic studies of the relation between Chlamydia pneumoniae and atherosclerosis: Does study design influence results? Am Heart J 2003;145:409-417.

16. Di Tano G, Picerno I, C alisto ML, D elia SA, Lagana P, Spataro P. [C hlamydia pneumoniae and Helicobacter pylori infections in acute myocardial infarction]. Ital Heart J Suppl 2000;1(12):1576-1581. 17. Alpert JS, Thygesen K, Antman E, Bassand JP. Myocardial infarction redefined-A consensus document of The Joint European Society of Cardiology/American College of $\mathrm{C}$ ardiology $\mathrm{C}$ ommittee for the Redefinition of Myocardial Infarction. J Am Coll Cardiol 2000;36:959969 .

18. 0 besity: Preventing and managing the global epidemic. Report of a W HO Consultation. Ginebra:W HO ; 2000.W HO Technical Report Series.

19. Grayston JT,W ang SP, Kuo CC, C ampbell LA. C urrent knowledge on Chlamydia pneumoniae, strain TW AR, an important cause of pneumonia and other acute respiratory diseases. Eur J C lin Microbiol Infect D is 1989;8:191-202.

20. Mendall MA, C arrington D, Strachan D, Patel P, Molineaux N, Levi J et al. Chlamydia pneumoniae: Risk factors for seropositivity and association with coronary heart disease.J Infect 1995;30:121-128.
21. Patel P, Mendall MA, Carrington D, Strachan DP, Leatham E, Molineaux $\mathrm{N}$ et al.Association of Helicobacter pylori and Chlamydia pneumoniae infections with coronary heart disease and cardiovascular risk factors. BMJ 1995;311:711-714.

22. Mizooka M, Ishikawa S. JMS C ohort Study Group. Prevalence of Chlamydia pneumoniae in Japanese rural districts; association of smoking and physical activity with Chlamydia pneumoniae seropositivity. Intern Med 2003:42: 960-966.

23. Koh W P,Taylor MB, Chew SK, Phoon MC, Kang KL, Chow VT. Chlamydia pneumoniae IgG seropositivity and clinical history of ischemic heart disease in Singapore. J Microbiol Immunol Infect 2003;36:169-174. 24. Buja LM,W illerson JT.The role of coronary artery lesions in ischemic heart disease: Insights from recent clinicopathologic, coronary arteriographic, and experimental studies. Human Pathol 1987;18:451-461. 25. Kuo CC, Shor A, Campbell LA, Fukushi H, Patton DL, Grayston JT. Demonstration of Chlamydia benefit from antibiotic treatment concerning embolus pneumoniae in arteriosclerotic lesions of coronary arteries. I Infect $D$ is 1993;167: 841-849.

26. Campbell LA, 0 'Brien ER, C appuccio AL, Kuo CC, W ang SP, Stewart $D$ et al. Detection of Chlamydia pneumoniae TW AR in human coronary atherectomy tissue. I Infect D is 1995;172: 585-588.

27. W ald N J, Law MR, Morris JK, Z hou X, W ong Y,W ard ME. Chlamydia pneumoniae infection and mortality from ischaemic heart disease: Results from a large prospective study. BMJ 2000;321: 204-207. 28. Siscovick DS, Schwartz SM, Corey L, G rayston JT, A shley R, W ang SP. Chlamydia pneumoniae, herpes simplex virus type 1 , and cytomegalovirus and incident myocardial infarction and coronary heart disease death in older adults:The $\mathrm{C}$ ardiovascular 